\title{
Beast of burden? Understanding the impact of outpatient total hip and knee replacement on caregivers at home
}

\author{
Braeden M. Page, BKin (1) - David R. Urbach, MD, MSc, FRCSC, FACS • \\ Jesse I. Wolfstadt, MD, MSc, FRCSC • Natalie Clavel, MD, MHSc, FRCPC • \\ Richard Brull, MD, FRCPC
}

Received: 23 August 2021/Revised: 28 September 2021 / Accepted: 4 October 2021/Published online: 1 November 2021

(C) Canadian Anesthesiologists' Society 2021

Keywords ambulatory surgery $\cdot$ caregiver burden . informal caregiver · total hip arthroplasty .

total knee arthroplasty

Recent advances in surgical technique, preoperative assessment, patient preparation, anesthesia, and analgesia have all contributed to the ability to perform same-day total hip arthroplasty (THA) and total knee arthroplasty (TKA) in select patients. ${ }^{1}$ Traditionally, patients undergoing THA or TKA would have stayed in hospital for nearly five days after surgery for pain management and rehabilitation. These patients occupied valuable hospital beds that could be used by other services and cost the healthcare system tens of thousands of dollars per hospital stay. ${ }^{2}$ The introduction of targeted motor-sparing nerve blocks, novel perineural adjuncts, and local anesthetic infiltration techniques, all combined with preventative and therapeutic perioperative multimodal analgesia and management of

B. M. Page, BKin ( $ه)$

Women's College Hospital Research Institute, Women's College

Hospital, Toronto, ON, Canada

e-mail: Braeden.Page@wchospital.ca

Institute of Medical Science, University of Toronto, Toronto, ON, Canada

D. R. Urbach, MD, MSc, FRCSC, FACS

Department of Surgery, Temerty Faculty of Medicine,

University of Toronto, Toronto, ON, Canada

Department of Surgery, Women's College Hospital, Toronto,

ON, Canada

J. I. Wolfstadt, MD, MSc, FRCSC

Department of Surgery, Temerty Faculty of Medicine,

University of Toronto, Toronto, ON, Canada related side effects, has led to reductions in the length of stay such that an ever-increasing cohort of patients is being discharged from the hospital on the same day as surgery. ${ }^{3}$ The current COVID-19 pandemic has further accelerated interest in shorter-stay total joint arthroplasty (TJA) surgery to relieve the strain on our healthcare system and the significant surgical backlog. ${ }^{4}$

In addition to favourable patient outcomes, including superior quality of recovery, ${ }^{1}$ performing THA and TKA procedures on an outpatient basis results in significant reductions in hospital costs. One Canadian study found the total median cost for an outpatient TKA to be CAD 6,487.50 per patient, corresponding to an approximate median cost savings of $30 \%$ compared with an inpatient TKA, which has a median cost of CAD 9,643.06 per patient. ${ }^{5}$ These savings largely stem from reductions in medication, nutrition, and nursing care costs. ${ }^{5}$ While the transition from traditional inpatient to outpatient surgery offers significant cost savings to the Canadian healthcare system, the extent to which the burden of postoperative

Granovsky Gluskin Division of Orthopaedics, Sinai Health, Toronto, ON, Canada

N. Clavel, MD, MHSc, FRCPC

Department of Anesthesiology \& Pain Medicine, Temerty Faculty of Medicine, University of Toronto, Toronto, ON, Canada

R. Brull, MD, FRCPC

Women's College Hospital Research Institute, Women's College Hospital, Toronto, ON, Canada

Department of Anesthesiology \& Pain Medicine, Temerty Faculty of Medicine, University of Toronto, Toronto, ON, Canada 
care for THA and TKA patients has been transferred onto informal caregivers is unknown.

Informal caregivers have been referred to as the "invisible backbone" of the healthcare system, yet there is a lack of awareness and understanding of the scale of their contribution in caring for patients undergoing major ambulatory surgery. ${ }^{6}$ Though the caregiving process can be rewarding, there are important challenges and stressors associated with the caregiving experience. Caregiver burden refers to the response to emotional, financial, physical, psychological, and social stressors incurred while caring for another individual. ${ }^{7}$ Existing evidence examining caregiver burden almost exclusively focuses on informal caregivers caring for individuals dealing with chronic diseases in an outpatient setting, such as dementia. In these settings, it has been shown that one in three caregivers will suffer from depression, ${ }^{8}$ while high levels of stress and anxiety, social isolation, and physical and financial strain are commonplace. ${ }^{7,9-12}$ Nevertheless, these findings may not be generalizable to the perioperative setting, especially for major orthopedic surgery. Indeed, the natural progression of caregiver burden in chronic disease follows a crescendo pattern, with the burden of care gradually increasing as a patient's condition deteriorates. Conversely, the progression of caregiver burden in a limited, self-resolving episode of care such as elective outpatient surgery has a decrescendo pattern. These caregivers enter the process with the burden of care at its peak; the burden declines and ultimately resolves once the patient is rehabilitated and no longer requires caregiver assistance.

Our current understanding of caregiver burden following major elective orthopedic surgery is limited. With $>120,000$ primary TJAs performed annually in Canada $^{1}$ and a growing emphasis on outpatient surgery, more caregivers are being asked to perform activities at home that would be typically be carried out by a healthcare professional in a hospital. There is very little evidence examining the burden of care in the outpatient TJA setting. Only one study has examined the perspectives of caregivers following outpatient TJA. Churchill et al. aimed to gain insight into the advantages and disadvantages of same-day discharge from the perspective of both the patient and caregiver. ${ }^{13}$ The authors identified several unique considerations to improve the experience of same-day discharge, specifically ensuring both patient and caregiver are present when receiving postoperative education as caregivers noted added stress and confusion when important health-related information was missing or not well understood. ${ }^{13}$ Nevertheless, the results from this study may not be reliable and valid because of a limited sample size of only eight outpatient THA caregivers. Likewise, caregivers were recruited to participate in the study at 3 and 6 months postoperatively, potentially limiting the caregivers' ability to recall the caregiving experience and minimizing the factors that contributed to the burden of care.

Following years of preparation and development, our country's first exclusively outpatient total joint arthroplasty program was launched in March 2018 and based at Women's College Hospital, an independent free-standing ambulatory institution in Toronto. Central to the success of our program was the leadership role of the anesthesiology department within all phases of the patients' perioperative journey. ${ }^{14,} 15$ Having performed nearly 500 outpatient TJAs to date (280 THA, 209 TKA), we have gained a fulsome understanding of nearly all key stakeholders' needs and experiences, including patients, surgeons, anesthesiologists, nurses, physiotherapists, pharmacists, and hospital administrators. Nevertheless, what remains poorly defined and perhaps inadvertently overlooked are the needs and experiences of the final key stakeholder, the informal caregivers who provide care to our outpatients.

Integral to the development, implementation, and execution of a safe and successful same-day TJA perioperative program, the entire perioperative team, which includes the complementary skill sets of anesthesia, surgery, nursing, physiotherapy, occupational therapy, and social work, is uniquely positioned to examine caregiver burden and inform best practice. Similar to the perioperative surgical home model, ${ }^{16,}{ }^{17}$ an anesthesiologist-led interdisciplinary team that approaches the perioperative episodes of care as one continuum rather than exclusive stages, and extending well into the post-acute rehabilitation phase, is best suited to ensure that our informal caregivers are adequately supported and that their unmet needs are recognized with a view towards improvement. Based on our goals of identifying, quantifying, and understanding the actionability of the various factors contributing to caregiver burden, we propose the following biopsychosocial framework as a guide for future research and to help clinicians, healthcare systems, and policy makers think about the impact of the shift to more outpatient surgery on caregivers (Figure). Our proposed framework is divided into six domains, each containing a variety of factors that contribute to the overall burden of care experienced by the caregiver. Our framework and domains were created based on our own experience from our outpatient THA and TKA program at Women's College Hospital and drawn from our review of the generalizable findings within the dementia-related literature and the very limited literature in the outpatient TJA setting. ${ }^{7-13,} 18$ Through informal discussions with patients and caregivers from the preoperative assessment 


\section{Caregiver Burden Framework}

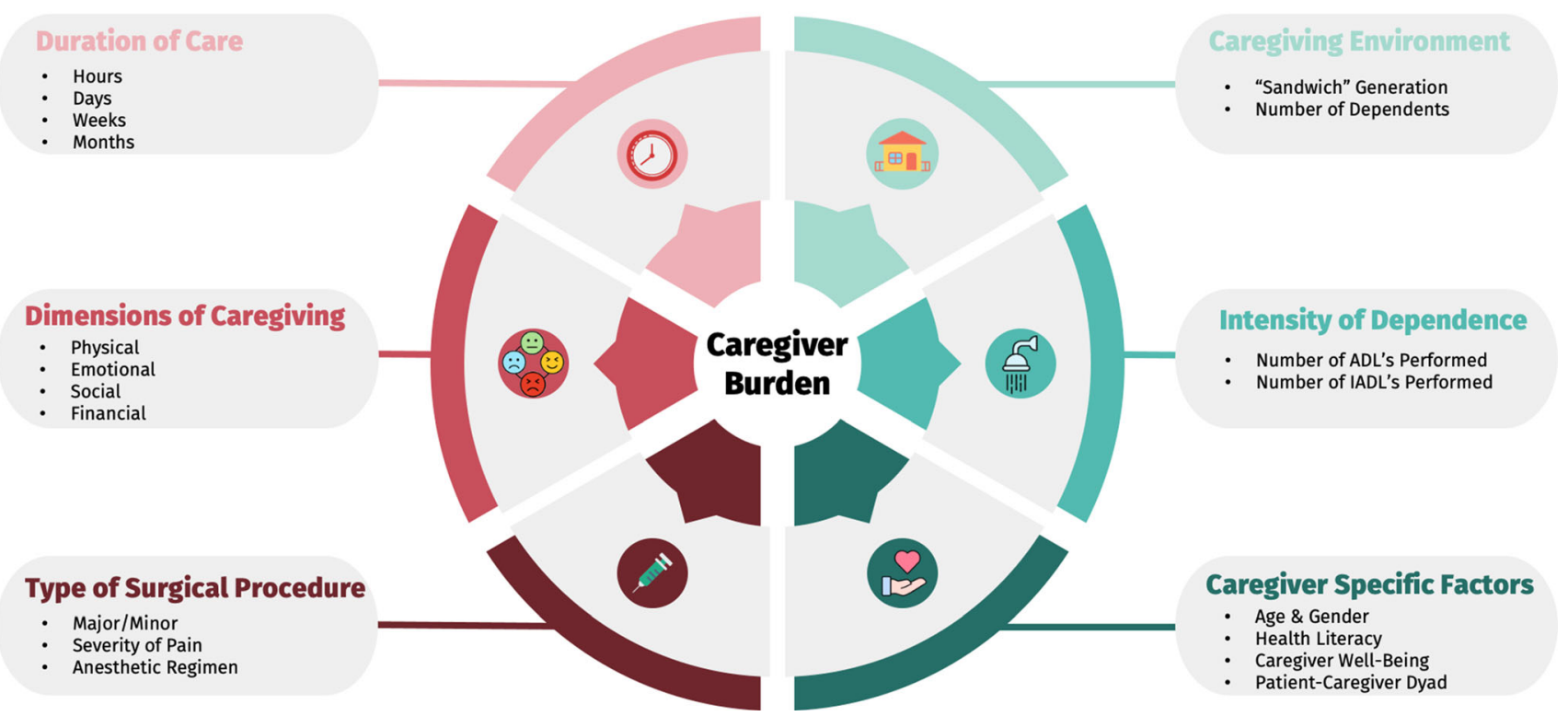

Figure Caregiver burden framework identifying the various factors that may contribute to the burden of care experienced by informal caregivers in the outpatient setting.

clinic to virtual care recovery at home through video conferences as part of our quality insurance program, all in combination with nursing and physiotherapy feedback, we are continuously developing an understanding of the different aspects of the burden of care among our informal caregivers.

The first domain is the duration of care, that is, how long the caregiver is providing care for the patient. The intensity of dependence domain considers how many activities of daily living and instrumental activities of daily living the caregiver is performing/assisting the patient with. The third domain involves the type of surgical procedure, as different procedures may require different caregiving expectations and may elicit varying pain responses in the patient according to the nature of the procedure and analgesic regimen. The dimensions of caregiving domain assesses the physical, emotional, social, and financial strain placed on the caregivers. Caregiving environment examines the number of dependents of the caregiver, as many caregivers are part of the "sandwich" generation, providing care for both elderly parents and their own children. ${ }^{19}$ The final domain contains caregiver-specific factors, such as the caregiver's well-being, age, gender, relationship to the patient, and health literacy (the ability to understand and use healthcare information).
It is important to note the limitations within our framework. Our framework and domains were designed based on our experience at a single center, which may not be generalizable to other institutions. Furthermore, our current framework may not capture all factors contributing to caregiver burden. Indeed, our framework is neither definitive nor validated; nevertheless, we believe that it serves as a practical foundation to bring caregiver burden into focus and guide future research. As the number of traditional inpatient surgical procedures transitioning to an outpatient setting continues to evolve and expand, so too will our proposed framework in order to elucidate the impact of this transition on informal caregivers. Using our framework, we aim to take inventory of the factors and dimensions contributing to caregiver burden within our ambulatory TJA program and identify the most important factors contributing to the burden of care at various milestones along the perioperative journey with a view towards intervention, mitigation, and possibly prevention.

Finally, with over half of TJA procedures expected to be performed in the outpatient setting by the year $2026,{ }^{20}$ and a surge in TJA volumes as hospitals attempt to work through the backlog of postponed surgeries due to COVID$19,{ }^{21,22}$ caregiver burden following outpatient surgery has the potential to become an important societal problem. As more procedures shift to the outpatient setting, more 
responsibilities are being placed onto caregivers earlier in the recovery pathway. Not only are we figuratively and likely literally passing the buck, but the added strain placed on informal caregivers may inadvertently create an entirely new population of sufferers who may require medical care. Anesthesiologists, alongside the entire perioperative care team, will continue to lead accelerated recovery pathways within novel ambulatory programs; nevertheless, we must also endeavour to comprehensively explore and examine the experiences of informal caregivers to understand and ultimately address the most important factors contributing to their burden.

Acknowledgements This reflection contributes to the partial fulfilment of the requirements for the Master of Science in Medical Science at the University of Toronto, Toronto, Ontario, for Braeden M. Page. Braeden M. Page acknowledges Women's College Research Institute for providing support during the completion of his degree.

\section{Disclosures None.}

Funding statement Braeden M. Page is supported by the Canadian Institutes of Health Research (CIHR) through the Frederick Banting and Charles Best Canada Graduate Scholarship - Master's (CGS-M). Richard Brull receives research time support from the Evelyn Bateman Cara Operations Endowed Chair in Ambulatory Anesthesia and Women's Health, Women's College Hospital, and Merit Award Program, Department of Anesthesia and Pain Medicine, Toronto, ON, Canada.

Editorial responsibility This submission was handled by Dr. Philip M. Jones, Deputy Editor-in-Chief, Canadian Journal of Anesthesial Journal canadien d'anesthésie.

\section{References}

1. Bodrogi A, Dervin GF, Beaulé PE. Management of patients undergoing same-day discharge primary total hip and knee arthroplasty. CMAJ 2020; 192: E34-9.

2. Canadian Institute for Health Information. Hip and knee replacements in Canada, 2014-2015: Canadian Joint Replacement Registry Annual Report. Ottawa, ON; 2017. Available from URL: https://secure.cihi.ca/free_products/cjrrannual-report-2016-en.pdf (accessed October 2021).

3. Stambough JB, Nunley RM, Curry MC, Steger-May K, Clohisy $J C$. Rapid recovery protocols for primary total hip arthroplasty can safely reduce length of stay without increasing readmissions. J Arthroplasty 2015; 30: 521-6.

4. Gleicher Y, Peacock S, Peer M, Wolfstadt J. Transitioning to outpatient arthroplasty during COVID-19: time to pivot. CMAJ 2021; DOI: https://doi.org/10.1503/cmaj.78145.

5. Huang A, Ryu JJ, Dervin G. Cost savings of outpatient versus standard inpatient total knee arthroplasty. Can J Surg 2017; 60: 57-62.
6. Levine $C$. Putting the spotlight on invisible family caregivers. JAMA Intern Med 2016; 176: 380-1.

7. Etters L, Goodall D, Harrison BE. Caregiver burden among dementia patient caregivers: a review of the literature. J Am Acad Nurse Pract 2008; 20: 423-8.

8. Sallim AB, Sayampanathan AA, Cuttilan A, Chun-Man Ho R. Prevalence of mental health disorders among caregivers of patients with Alzheimer disease. J Am Med Dir Assoc 2015; 16: 1034-41.

9. Pinquart $M$, Sörensen $S$. Differences between caregivers and noncaregivers in psychological health and physical health: a meta-analysis. Psychol Aging 2003; 18: 250-67.

10. Torti FM Jr, Gwyther LP, Reed SD, Friedman JY, Schulman KA. A multinational review of recent trends and reports in dementia caregiver burden. Alzheimer Dis Assoc Disord 2004; 18: 99-109.

11. Cheng ST. Dementia caregiver burden: a research update and critical analysis. Curr Psychiatry Rep 2017; DOI: https://doi.org/ 10.1007/s11920-017-0818-2.

12. Kršková T, Zeleniková R. Association between burden and depresion in caregivers of dementia patients. Cent Eur J Nurs Midw 2018; 9: 866-72.

13. Churchill L, Pollock M, Lebedeva Y, et al. Optimizing outpatient total hip arthroplasty: perspectives of key stakeholders. Can J Surg 2018; 61: 370-6.

14. Favaro A, St. Philip E. Home in six hours: Toronto hospital offers same-day joint replacement surgery. CTV National News. April 2018. Available from URL: https://www.ctvnews.ca/canada/ home-in-six-hours-toronto-hospital-offers-same-day-jointreplacement-surgery-1.3881534 (accessed October 2021)

15. Boyle T. He got his knee replaced at a Toronto hospital. A few hours later he was climbing stairs. Toronto Star. April 2018. Available from URL: https://www.thestar.com/news/gta/2018/04/ 11/he-got-his-knee-replaced-at-a-toronto-hospital-a-few-hourslater-he-was-climbing-stairs.html (accessed October 2021).

16. American Society of Anesthesiologists. Perioperative surgical home (PSH) overview. Available from URL: https://www.asahq. org/psh/aboutpsh/an-overview (accessed October 2021).

17. Kain ZN, Vakharia S, Garson L, et al. The perioperative surgical home as a future perioperative practice model. Anesth Analg 2014; 118: 1126-30.

18. Zomar BO, Bryant DM, Marsh JD, Lanting BA. Assessment of informal caregiver assistance and strain with total hip and knee arthroplasty. J Arthroplasty 2021; 36: 2424-30.e1.

19. Steiner AM, Fletcher PC. Sandwich generation caregiving: a complex and dynamic role. J Adult Dev 2017; 24: 133-43.

20. Van Horne A, Van Horne J. Patient-optimizing enhanced recovery pathways for total knee and hip arthroplasty in Medicare patients: implication for transition to ambulatory surgery centers. Arthroplast Today 2019; 5: 497-502.

21. Wang J, Vahid S, Eberg M, et al. Clearing the surgical backlog caused by COVID-19 in Ontario: a time series modelling study. CMAJ 2020; 192: E1347-56.

22. Arthritis Society. The wait: Addressing Canada's critical backlog of hip and knee replacement surgeries. June 2021. Available from URL: https://arthritis.ca/getmedia/63f2646b-0c7a-4064-98cfc1ac8460c326/ArthritisSociety_WaitTimesReport_EN.pdf (accessed October 2021).

Publisher's Note Springer Nature remains neutral with regard to jurisdictional claims in published maps and institutional affiliations. 